\title{
UN NUEVO DISEÑO DE TRAMPA DE CAÍdA PARA EL ESTUDIO DE POBLACIONES DE COLEÓPTEROS TERRESTRES DE SUPERFICIE
}

\author{
A. de los Santos ${ }^{1}$, C. Montes ${ }^{1}$, L. Ramírez-Díaz ${ }^{2}$.
}

\begin{abstract}
A new pitfall trap for studying soil surface coleoptera, simple and inexpensive, is described. The new trap is really useful for cuantitative studies of mixed populations of soil surface coleoptera.
\end{abstract}

The efficiency of this pitfall trap was field tested.

Entre las técnicas más utilizadas para muestrear poblaciones de artrópodos terrestres de la superficie del suelo, están las trampas de caída (Greenslade, 1964). Consisten, en recipientes que se sitúan en un lugar apropiado y se nivelan con la superficie del suelo. Los individuos en actividad caen en su interior al realizar sus desplazamientos.

Cuando se estudian simultáneamente comunidades de coleópteros de superficie con trampas de caída convencionales junto con otras técnicas (recolección estandarizada), se encuentran importantes discrepancias en los resultados, respecto a la estructura cualitativa de la comuni-

1.--Departamento de Zoología. Universidad de Murcia.

2.-Departamento de Ecología. Universidad de Murcia. 
dad (Kirk, 1971). Se pone de manifiesto la existencia de un importante número de factores independientes de la densidad, que intervienen, de una forma conjunta, sobre la eficiencia de la técnica que utiliza trampas de caída.

De una forma general, los factores más importantes que tienen relación con la eficiencia de las trampas, son los referentes a eficacia en la captura y retención de los individuos capturados, predación en el interior de la trampa, uso de conservadores, alteración del suelo en los procesos de colocación y vaciado, inundación por el agua de lluvia y facilidad de utilización.

Se han ideado una gran variedad de trampas de caída (en cuanto a diferentes tamaños, formas y materiales utilizados), prácticamente, existen tantos diseños como investigadores han empleado la técnica, pero la mayor parte de ellos no son más que modificaciones de uno o dos modelos básicos ya existentes. Todos estos modelos están pensados para operar con una determinada población o grupo de poblaciones (generalmente con familias) con características morfológicas, ecológicas y de comportamiento más o menos similares. Realmente, no existe ningún modelo o diseño que sirva para cuantificar, de una forma eficaz, comunidades de coleópteros más o menos diversificadas y heterogéneas, en cuanto a tamaños, formas, comportamiento, grado de movilidad, requerimientos ecológicos, capacidad esporádica de vuelo, etc., de las especies.

Durante el estudio de la estructura y dinámica de una comunidad de coleópteros terrestres en dos ecosistemas característicos del Bajo Guadalquivir (pastizal de terófitos efímeros y pinar mediterráneo) se utilizó un nuevo diseño de trampa de caída, que reúne las ventajas de una serie de modelos ya existentes. De esta manera, se pudo operar, de una forma eficaz, con toda la comunidad (de los Santos, 1983).

El nuevo modelo, al que se ha denominado «Sistema de embudos compartimentados», se basa en la utilización combinada de varias estructuras en forma de embudo (Figura 1).

El objetivo principal de este sistema es el de facilitar y asegurar la captura del mayor número de individuos que se desplazan por una superficie determinada de suelo. 


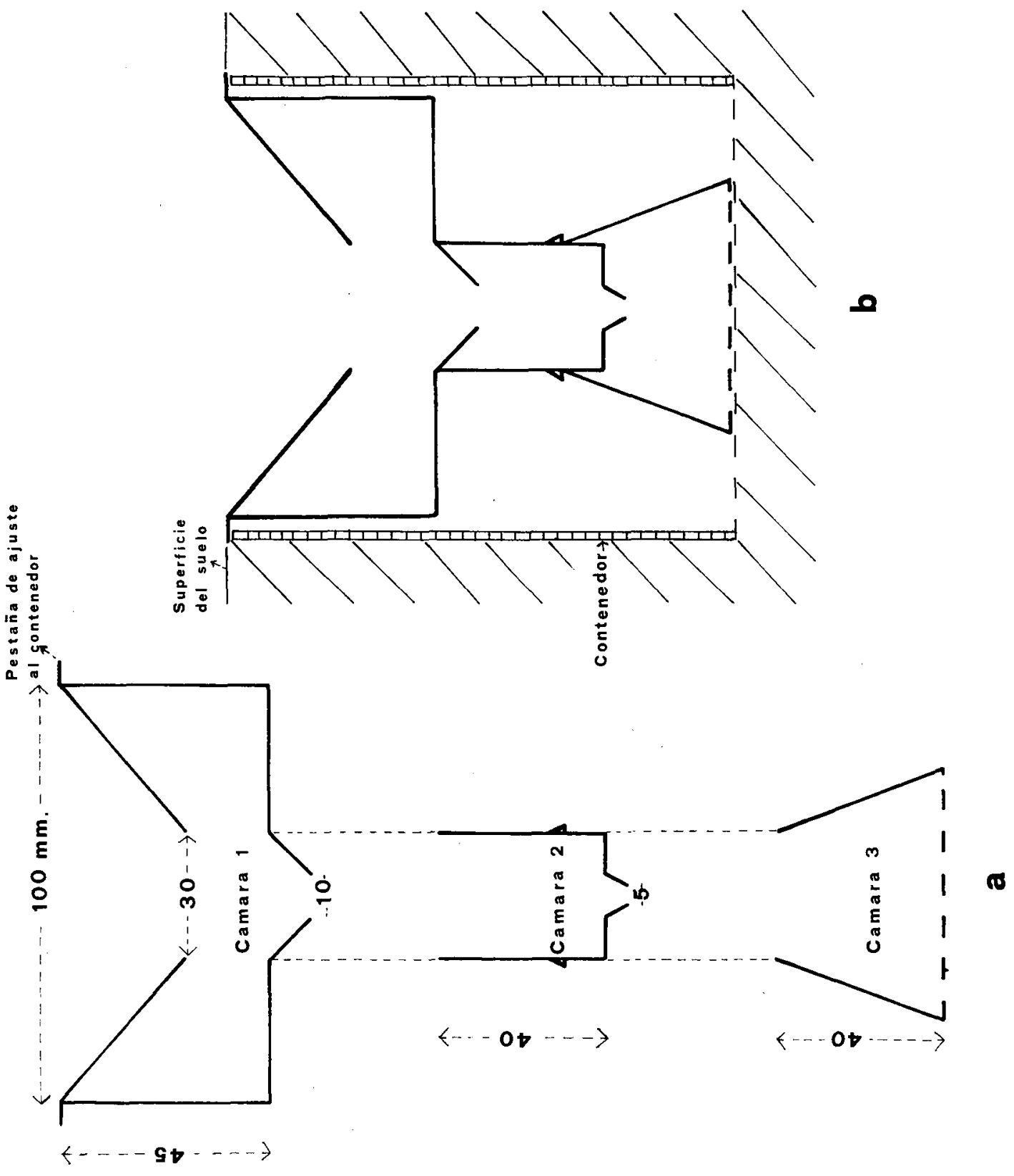

Figura 1.-Diagrama de la nueva trampa de caída.

a._Dimensiones de cada una de las cámaras.

b.-Estructuras acopladas en el suelo. 
El modelo está compuesto por tres cámaras superpuestas, comunicadas entre sí por embudos de diámetro cada vez más pequeño (Figura 1. a). Éstos, facilitan y seleccionan el paso de individuos de diferente tamaño a cada una de las cámaras, asegurando su retención (Wojcik y otros, 1972). Los diámetros de cada embudo $(30 \mathrm{~mm}$., $10 \mathrm{~mm}$., y $5 \mathrm{~mm}$.) se han ajustado a los tres tipos generales de tamaños en que se puede englobar la mayoría de las especies de coleópteros terrestres (Czechowski, 1979). De esta forma, la cámara superior atrapa las especies grandes e impide la entrada de otros animales, principalmente micromamíferos, que podrían destrozar la captura (Pausch y otros, 1979). La base del cono de recepción tiene el diámetro convencional de $10 \mathrm{~cm}$. (Luff, 1975). El fondo de esta cámara, así como el de la siguiente, consiste en una plataforma donde los coleṕteros que, por su tamaño, no pueden pasar a la cámara inferior, puedan desplazarse y dejar libre el orificio para la caída de los individuos más pequeños. Éstos, tienden a pasar a las cámaras inferiores, bien por caída libre o por la tendencia geófila que presentan en su comportamiento de huida.

La última cámara tiene su fondo perforado para drenar el agua de lluvia que puede caer sobre la trampa e inundarla.

Todo el sistema se ajusta a un contenedor metálico perforado en su base e introducido a ras del suelo, de una forma definitiva, durante todo el período de estudio (Figura 1. b). Este contenedor facilita la utilización de la trampa (colocación y vaciado) sin alterar la estructura del suelo (Mejer, 1972).

Los materiales que pueden utilizarse para construir las distintas cámaras pueden ser variados. Por lo general, pueden quedar dependientes del tipo de recipientes de que se disponga, se ajusten a este modelo y se tengan disponibles. Por ejemplo, como primera cámara resulta muy eficaz el empleo de mosqueros tradicionales de vidrio y, como segunda y tercera cámaras, se pueden utilizar recipientes de plástico, variados, que existen en el mercado. En este caso, hay que resaltar que el ajuste del borde de la cámara superior con el contenedor debe ser perfecto, de tal manera que no queden grietas entre ambos recipientes.

Para evaluar la eficiencia de la nueva trampa, se colocaron dos filas de 12 unidades de muestreo. Una con embudos compartimentados y otra con trampas tradicionales de igual diámetro de entrada. Ambas 
se situaron, durante una semana (23 al 30 de Enero de 1982), en una franja de suelo cubierta de hojarasca. Los resultados se muestran en la tabla 1. En ella, se indican los estadísticos de la densidad relativa de las especies de coleópteros terrestres más importantes capturados durante ese período de estudio por los dos tipos de trampas utilizadas.

Asímismo, y con objeto de evaluar diferencias en la captura, según distintos tipos de comportamientos y tamaños de las especies, se calcularon los mismos estadísticos para las dos familias más importantes que se capturaron y para los dos tipos distintos de tamaño. La comparación estadística de las muestras se realizó mediante el test de la U de Mann-Whitney (Siegel, 1956), cuyos resultados se muestran en la tabla 1.

Se observa como existen diferencias significativas en la captura por los dos tipos de trampas para las especies pequeñas (Mastigus palpalis Latr., Paromalus flavicornis Herbst. y Brachinus bellicosus Duf.), pero no para las de tamaño grande (Pterostichus (Steropus) globosus ebenus Quens.) ya que éstas, debido a su peso, no pueden escapar de los sistemas convencionales de trampas de caída. Esto mismo se detecta cuando se comparan de una forma global los dos tipos de tamaño de individuos recolectados.

Respecto a la eficacia en la captura para grandes grupos funcionales, la nueva trampa retiene eficazmente a los individuos de la familia Curculionidae (fitófagos), conocidos como buenos trepadores. Asímismo, existen diferencias en la captura para la familia Carabidae (básicamente depredadores), caracterizada por englobar una gran riqueza de especies con diferentes tamaños y comportamientos. Esta diferencia será más o menos acusada dependiendo del tamaño de las especies más frecuentes y dominantes. Así, en este caso, la familia Carabidae, considerada globalmente, no presenta una diferencia tan marcada como Curculionidae, ya que, durante el período de estudio, Pterostichus (Steropus) globosus ebenus (tamaño grande) fue la especie dominante. De todas formas, para zonas de estudio heterogéneas y períodos de trabajo prolongados, las diferencias en la captura son muy notables (de los Santos, 1983).

Así pues, podemos concluir que la nueva trampa «Sistema de embudos compartimentados» resulta enormemente eficiente para los estu- 
Tabla 1 .- Estimas de la densidad relativa y valores de U (test Mann- Whitney) de varias especies, familias y tamaños de coleópteros terrestres de una banda de suelo cubierto de hojarasca.

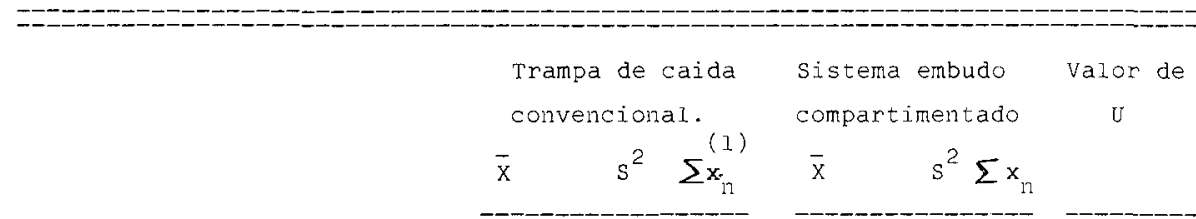

ESPECIES

$\begin{array}{lrrrrrrr}\text { Pterostichus globosus Quens. } & 7,1 & 25,5 & 84 & 6,25 & 12,9 & 75 & 72,2 \\ \text { Brachinus bellicosus Duf. } & 1,0 & 4,0 & 12 & 5,91 & 100,3 & 71 & 35,5 * \\ \text { Mastigus palpalis Latr. } & 0,5 & 0,8 & 6 & 2,25 & 9,6 & 27 & 36,5 * \\ \text { Paromalus flavicomis Herbst. } & 2,1 & 6,8 & 25 & 5,50 & 20,6 & 66 & 33,5 *\end{array}$

FAMIIIAS

Carabidae

Curculionidae

$8,2 \quad 36,6$

9915,00

$147,4 \quad 180$

35,0

$\begin{array}{llllll}0,7 & 6,7 & 9 & 1,8 & 2,8 & 22\end{array}$

$33,0 *$

TAMAÑOS

Pequeños $(<10 \mathrm{~mm}$. )

$\begin{array}{llllll}4,1 & 12,6 & 49 & 18,58 & 184,8 & 223\end{array}$

$1.2,5$

Grandes $(>10 \mathrm{~mm}$.

$8,1 \quad 30,9$

97

8,66

$17,1 \quad 104$

66,0

* $\mathrm{P}<0,05 ; \quad \mathrm{P}<0,001 ; \quad \mathrm{n}_{1}=\mathrm{n}_{2}=12$

( 1 ) $\sum x_{n}=$ Número total de individuos capturados en las $n$ trampas.

dios de descripción de la estructura cualitativa y cuantitativa de comunidades de coleópteros terrestres de la superficie del suelo. Al no utilizar conservador se evitan los efectos de exterminio de las poblaciones, cuando se opera en períodos prolongados, así como las interacciones de atracción y repulsión que provoca (Luff. 1968).

El sistema de embudos superpuestos en gradiente de tamaño, evita la fuga de las especies trepadoras y voladoras, así como los efectos de predación entre la captura.

Por su sistema de drenaje evita los fenómenos de microclima que se produce cuando se utilizan techos que impiden la inundación de las trampas. 


\section{BIBLIOGRAFÍA}

CZECHOWSKI W., 1979. Sampling of Carabidae (Col.) by Barber's traps and biocenometric method in urban environment. Bull. Acad. Sci. Pol. Ser. Sci. Biol., 27 (6): 461-465.

GREENSLADE P. J. M., 1964. Pitfall trapping as a method for stying populations of Carabidae (Col.). J. Anim. Ecol., 33: 301-310.

KIRK V. M. 1971. A field insectary for ground beetles (Col. Carabidae). Ann. Entomol Soc. Amer., 64: 302-303.

LUFF M. L. 1968. Some effects of formalin on the numbers of Coleoptera caugt in pitfall traps. Ent. mon. Mag., 104: 115-116.

LUFF M. L. 1975. Some features influencing the efficiency of pitfalltraps. Oecologia (Berlín), 19: 345-357.

MAJER J. D. 1978. An improved pitfall trap for sampling ants and other epigaeic invertebrates. J. Aus. ent. Soc., 17: 261-262.

PAUSCH R. D.; ROBERTS S. J.; BARNEY R. J.; ARMBRUST E. J. 1979. Linear pitfall traps, a modification of an established trapping method. The great Lakes entomologist, 12 (4): 149-151.

SANTOS A. de los, 1983. Biología y ecología de algunas poblaciones de coleópteros terrestres en dos ecosistemas del Bajo Guadalqui$\operatorname{vir}(S . W$. España). Tesis Doctoral. Universidad de Murcia.

SIEGEL S., 1956. Non-parametric Statistics for the Behavioral Sciences. McGraw-Hill. London, 312 págs.

WOJCIK D. P.; BANKS W. A.; HICKS D. M., PLUMLEY J. L., 1972. A simple inexpensive pitfall-trap for collecting artropods. Fla. Entomol., 55: 115-116. 